\title{
Estudo comparativo do controle da qualidade de comprimidos de nimesulida complexada e não complexada com $\beta$-ciclodextrina
}

\section{Comparative study of quality control of complexed and non-complexed nimesulide tablets with $\beta$-cyclodextrin}

Recebido em: 01/05/2020 Aceito em: $23 / 09 / 2020$
Mariana de Oliveira JESUS*; Vitória Alves de MORAES*;

Bruno dos Santos LIMA; Paula dos Passos MENEZES

Universidade Federal de Sergipe, UFS. Av. Marechal Rondon, $s / n$,

Jardim Rosa Elze, CEP 49100-0000. São Cristóvão, SE, Brasil.

E-mail:paula.dp.menezes@gmail.com

*As autoras contribuíram igualmente no desenvolvimento deste trabalho

\section{ABSTRACT}

The quality control of medicines through physicochemical analysis is of great importance in ensuring the quality of drugs. Therefore, this study compared the quality of nimesulide tablets with $\beta$-cyclodextrin $\left(\right.$ Maxsulid $\left.^{\circledR}\right)$ and without $\beta$-cyclodextrin $\left(\right.$ Nisulid $\left.^{\circledR}\right)$ as a way to investigate if the current tests are suitable to dosage forms that have included complexes with $\beta$-cyclodextrin on their composition. Physicochemical assays were carried out following the Brazilian Pharmacopeia 6th edition for nimesulide tablets. Both drugs showed good results in most tests of physicochemical characterization. The drug content and dissolution tests were performed with quantitative determinations by High Performance Liquid Chromatography. The results of drug content showed that the standard methodology is inadequate to extract the drug from inclusion complex and to quantify it efficiently. On dissolution, both drugs were approved according to the requirements established in the Brazilian Pharmacopeia. However, Maxsulid ${ }^{\circledR}$ was the only one to obtain its minimum dissolution in $120 \mathrm{~min}$.

It is clear the importance of implementing analytical protocols appropriated to new technologies applied in the production of new medicines commercially available.

Keywords: quality control; $\beta$-cyclodextrin; nimesulide.

\section{RESUMO}

O controle da qualidade de sólidos por meio de ensaios físico-químicos apresenta grande importância na garantia da qualidade de medicamentos. Diante disto, este estudo teve como objetivo comparar a qualidade de comprimidos de nimesulida com $\beta$-ciclodextrina $\left(\right.$ Maxsulid ${ }^{\circledR}$ ) e sem $\beta$-ciclodextrina $\left(\right.$ Nisulid $\left.^{\circledR}\right)$ como forma de investigar se os testes vigentes se aplicam às formas farmacêuticas que contenham complexos 
de inclusão com ciclodextrinas em sua composição. Para tal, foram realizados ensaios físico-químicos descritos na Farmacopeia Brasileira $6^{\text {a }}$ edição para comprimidos de nimesulida. Frente ao exposto, ambos os medicamentos obtiveram resultados satisfatórios na maioria dos testes de caracterização físico-química. Os testes de doseamento e dissolução foram realizados por meio de determinações quantitativas utilizando a Cromatografia Líquida de Alta Eficiência. Os resultados do doseamento mostraram que a metodologia aplicada não está adequada para extrair o fármaco do complexo de inclusão e assim doseá-lo de maneira eficiente. No teste de dissolução, os dois medicamentos foram aprovados de acordo com os critérios estabelecidos na Farmacopeia Brasileira. Porém, Maxsulid ${ }^{\circledR}$ foi o único a obter sua totalidade dissolvida em 120 minutos. Dessa forma, fica clara a importância da implementação de protocolos analíticos adequados às novas tecnologias aplicadas na produção de medicamentos.

Palavras-chave: controle da qualidade; $\beta$-ciclodextrina; nimesulida.

\section{INTRODUÇÃO}

Nimesulida (4'-nitro-2'-fenoximetanosulfonanilida) [1] é uma sulfonanilida pertencente à classe dos anti-inflamatórios não esteroidais (AINE), seletiva para a inibição da COX-2, com ação anti-inflamatória, antipirética e analgésica, sendo altamente prescrita $(1,2)$. Esse fármaco pertence à classe II no Sistema de Classificação Biofarmacêutica (SCB), o qual é caracterizado por baixa solubilidade e alta permeabilidade (3).

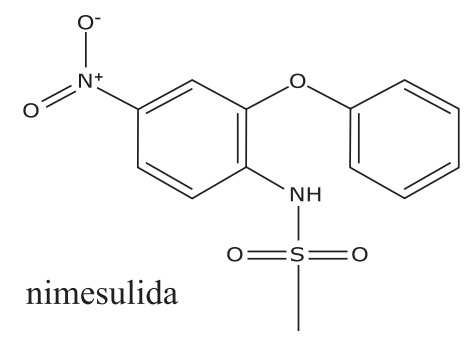

1

Dentre os excipientes disponíveis para melhorar a solubilidade desses fármacos existem as ciclodextrinas (CD), as quais têm um grande destaque no desenvolvimento de medicamentos, pela sua capacidade de aprimorar a estabilidade, biodisponibilidade e segurança de fármacos (4). Tratam-se de oligossacarídeos cíclicos que contêm uma cavidade central hidrofóbica e superfície externa hidrofílica, o que possibilita a formação de complexos de inclusão com fármacos e, assim, melho- ram as propriedades físico-químicas dos mesmos (5). Em suas formas naturais, as CD são obtidas em três conformações: $\alpha-C D, \beta-C D$ [2] e $\gamma$-CD, diferenciando-se pelo número de unidades de glicose (seis, sete e oito, respectivamente) (6).

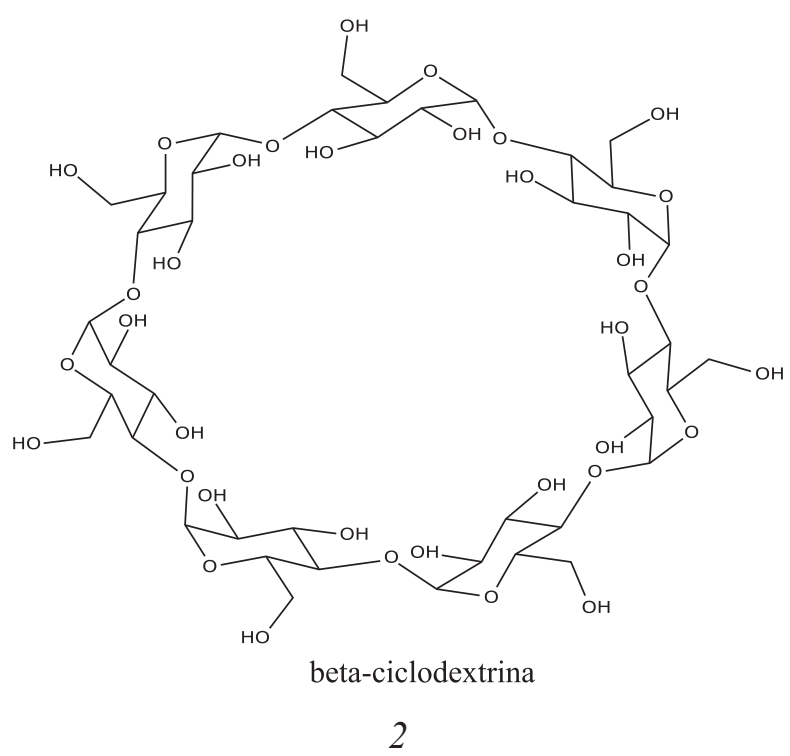

Para os fármacos mais hidrofóbicos, a complexação com as CD torna-se interessante, pois o caráter lipofílico da cavidade interior favorece a inclusão dos mesmos e a presença de hidroxilas livres na parte externa da estrutura facilita sua dissolução. Desse modo, as CD agem como transportadores moleculares, carreando fármacos hidrofóbicos até as membranas celulares, onde se dissociam e praticamente não são absorvidas devido ao seu elevado tamanho (4). 
A nimesulida possui um caráter fracamente ácido e baixa solubilidade em água, o que compromete a formulação para a via oral ou parenteral, podendo ter implicações em sua biodisponibilidade, fato que explica o interesse da indústria em sua associação com a $\beta$-CD (7).

A $\beta$-CD [2] é uma estrutura intermediária dentre as outras conformações das ciclodextrinas, formada por sete unidades de glicose. A $\beta$-CD é ideal para complexação devido ao tamanho da sua cavidade interna, boa eficiência de complexação, disponibilidade comercial e custo relativamente baixo, em relação às demais CDs (8).

$\mathrm{Na}$ maioria dos casos, a complexação de fármacos com as CD consegue melhorar o comportamento farmacológico dos mesmos no organismo (7). Exemplos disso são o estradiol, piroxicam e o anti-malárico artemisinina. No estudo de Schwarz e cols. (2017), o estradiol, em associação com a $\beta-C D$, pode ter sua solubilidade aumentada em mais de 100 vezes (9). O piroxicam, um AINE também complexado em $\beta-C D$, teve sua taxa de absorção aproximadamente 3,5 vezes mais rápida, comparada ao piroxicam livre $(10,11)$. A artemisina, quando complexada com $\gamma$-CD, teve sua biodisponibilidade aumentada, em média, 2,8 vezes em comparação a artemisina de referência (12).

No Brasil, assim como em muitos outros países, essa tecnologia de complexação tem se mostrado uma alternativa farmacêutica eficiente frente às limitações como solubilidade, biodisponibilidade e estabilidade. Todavia, na maioria das vezes, ainda é carente de regulamentações (13). A Farmacopeia Brasileira (FB), código oficial farmacêutico do país, regida pela Agência Nacional de Vigilância Sanitária (Anvisa), a qual descreve todos os ensaios de qualidade para medicamentos a fim de assegurar sua qualidade e segurança, não apresenta testes específicos para os fármacos complexados (14).

Diante desse contexto, esse estudo teve como objetivo comparar os testes de qualidade descritos

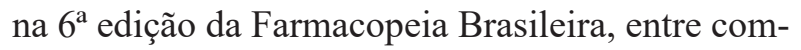
primidos de Nisulid $^{\circledR}$ (nimesulida do laboratório Aché) e comprimidos Maxsulid ${ }^{\circledR}$ (nimesulida associada à $\beta-C D$ da Farmasa), como forma de investigar se os testes vigentes aplicam-se às formas farmacêuticas que tenham complexos de inclusão na sua composição.

\section{MATERIAL E MÉTODOS}

Os reagentes utilizados na condução do presente estudo foram água ultrapura $\left(m i l i-Q^{\circledR}\right)$, acetonitrila grau HPLC (Honeywell/Riedel-de-Haën ${ }^{\circledR}$ ), polissorbato $80 \%$ (Dinâmica ${ }^{\circledR}$ ) e fosfato de potássio (Dinâmica ${ }^{\circledR}$ ). Para os ensaios de qualidade foram utilizados Nisulid $^{\circledR} 100 \mathrm{mg}$, lote 1810985 com validade abril de 2020, Maxsulid ${ }^{\circledR} 400 \mathrm{mg}$, lote B18J1014 com validade setembro de 2020 e nimesulida SQR (substância química de referência) cedida por uma farmácia de manipulação de Aracaju (SE).

Os equipamentos necessários para essa pesquisa foram Balança analítica Shimadzu ${ }^{\circledR}$, friabilômetro modelo 300 Nova Ética $^{\circledR}$, durômetro digital de bancada Nova Ética ${ }^{\circledR}$, modelo 299/ATTS, desintegrador Nova Ética ${ }^{\circledR}$, modelo 301/AC 03; cromatógrafo líquido de alta eficiência Shima$d z u^{\circledR}$; ultrassom ultracleaner 1400 Unique $^{\circledR}$; dissolutor Nova Ética ${ }^{\circledR}$ modelo 299/3 e agitador magnético com aquecimento Go-Stirrer $\left(\mathrm{Go}-\mathrm{Lab}^{\circledR}\right.$ ) modelo MS-H-S.

O presente estudo teve como abordagem a pesquisa quali-quantitativa, realizada por meio de procedimentos experimentais retratados nos volumes I de insumos farmacêuticos ativos (IFA) e Especiali-

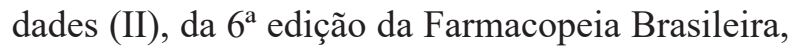
usando como base a monografia da nimesulida na forma farmacêutica de comprimidos (14). Todos os medicamentos em teste pertenciam aos mesmos lotes e os ensaios foram realizados entre os meses de agosto a novembro de 2019.

Determinação de peso. O teste de determinação de peso foi realizado a partir do método geral (5.1.1), descrito na Farmacopeia Brasileira, aplicada a forma farmacêutica de comprimidos sem revestimento, uma vez que não existem ressalvas para o referido teste no volume II da FB (14). Para a execução desse teste foram pesados 20 comprimidos individualmente, e a partir dos valores obtidos calculou-se a média de acordo com a Equação 1 (14):

$$
M=\frac{x_{1}+x_{2}+\cdots+x_{20}}{20}
$$

Equação 1 
Para serem aprovados no teste, a FB determina que no máximo, duas unidades estejam fora dos limites especificados, limites esses que variam de acordo com o peso médio resultante. Além disso, nenhuma unidade pode estar acima ou abaixo do dobro das porcentagens especificadas, a partir do peso médio aferido. No caso desta pesquisa, o limite usado foi $5 \%$ para ambos os medicamentos, de acordo com o peso médio determinado (14).

Friabilidade. Para execução do teste de friabilidade, foi utilizada a metodologia preconizada no método geral da Farmacopeia Brasileira (5.1.3.2). Para tal, foram pesados 20 comprimidos de Nisulid $^{\circledR}$ (pois o peso médio foi inferior a $0,65 \mathrm{~g}$ ) e $10 \mathrm{com}$ primidos de Maxulid ${ }^{\circledR}$ (peso médio superior a 0,65 g) e submetidos ao friabilômetro. Após 100 rotações do aparelho sob velocidade de 25 rotações por minuto, os comprimidos foram retirados. Em seguida, removidos possíveis resíduos de pó remanescentes e pesados novamente. A friabilidade foi medida pela porcentagem do pó perdido dos medicamentos, ou seja, pela diferença entre os pesos iniciais e finais. Para atender à especificação, essa perda deve ser igual ou inferior a $1,5 \%$ do seu peso (14).

Dureza. O ensaio de dureza foi realizado conforme descrito no método geral da FB (5.1.3.1). no qual os comprimidos ( $\mathrm{n}=10$ para ambas as especialidades farmacêuticas em análise) foram submetidos ao durômetro digital de bancada, a fim de aferir a força necessária para esmagá-los. De acordo com a FB, essa força deve ser medida em Newtons (N) e o resultado é informativo. A dureza foi determinada por meio da média das 10 medidas (14).

Desintegração. O ensaio de desintegração visa determinar se formas farmacêuticas sólidas do tipo comprimidos e cápsulas se desintegram dentro do limite de tempo especificado. Para esse teste, foi seguido o protocolo determinado na FB volume I (5.1.4.1), no qual foram adicionados seis comprimidos, acompanhados de um disco, em cada tubo das cestas do desintegrador. $\mathrm{O}$ equipamento operou com água destilada a $37^{\circ} \mathrm{C}$ como meio de imersão (volume em cada cuba $900 \mathrm{~mL}$ ), conforme preconizado. Para comprimidos não revestidos, como os investigados no presente estudo, o critério para a desintegração é de $30 \mathrm{~min}$, haja vista que não existe ressalva na monografia do medicamento. O tempo foi cronometrado e após $30 \mathrm{~min}$, o movimento do aparelho foi interrompido e observado se todos os comprimidos haviam desintegrado (14).

\section{Cromatografia líquida de alta eficiência} (CLAE). Para a quantificação do fármaco em estudo, a FB permite o uso da técnica de cromatografia líquida de alta eficiência (CLAE), de acordo com o método $\mathrm{B}$ da monografia da nimesulida, no teste de doseamento. Diante disto, CLAE-UV foi utilizada nas determinações quantitativas dos medicamentos. Para tal, foi utilizada, como fase estacionária, coluna C18 de fase reversa de $150 \mathrm{~mm}$ de comprimento; fase móvel: água e acetonitrila (50:50) e a detecção foi realizada em $\lambda=220 \mathrm{~nm}$, conforme preconizado (15).

De acordo com a RDC n ${ }^{\circ} 166$ de 2017, Art. $7^{\circ}$ "Os métodos analíticos compendiais devem ter sua adequabilidade demonstrada ao uso pretendido, nas condições operacionais do laboratório, por meio da apresentação de um estudo de validação parcial." Art. $8^{\circ}$ "A validação parcial deve avaliar, pelo menos, os parâmetros de precisão, exatidão e seletividade" (16).

Frente ao exposto, a validação parcial foi realizada conforme prevista na legislação vigente, a fim de atestar a confiabilidade do método utilizado (dados não apresentados no trabalho). Para atender aos critérios estabelecidos no Art. $8^{\circ}$ da RDC 166/2017 (16), foi realizada uma curva de calibração no intervalo linear de $10-100 \mu \mathrm{g} / \mathrm{mL}$, necessária para aferição da precisão e exatidão. A seletividade foi demonstrada a partir da resposta analítica.

Para obtenção da curva de calibração, as amostras foram preparadas, conforme descrito a seguir. Foram pesados $100 \mathrm{mg}$ de nimesulida SQR, os quais foram transferidos para um balão volumétrico de $100 \mathrm{~mL}$ para a obtenção de uma solução estoque de $1 \mathrm{mg} / \mathrm{mL}$. A mesma foi submetida a banho de ultrassom por 10 minutos. Em seguida, foram realizadas cinco diluições com fase móvel - água ultrapura e acetonitrila $50: 50(\mathrm{v} / \mathrm{v})$ - até as concentrações de 10, 25, 50, 75 e $100 \mu \mathrm{g} / \mathrm{mL}$. Estas soluções foram filtradas através de filtros de membrana politetrafluoretileno (PFTE) antes das análises no cromatógrafo. Todas as determinações foram realizadas em triplicata.

Dissolução. O teste de dissolução também foi conduzido de acordo com a monografia da nime- 
sulida. O procedimento foi realizado em um dissolutor utilizando o aparato pá, sob velocidade de agitação de $75 \mathrm{rpm}$. Nas cubas, foram adicionados $900 \mathrm{~mL}$ do meio de dissolução [tampão fosfato de potássio $\mathrm{pH} 7,4$ com polissorbato 80 a $2 \%(\mathrm{v} / \mathrm{v})]$. Todo o experimento foi realizado sob temperatura controlada de $37{ }^{\circ} \mathrm{C} \pm 1$ e em cada cuba foi adicionado um comprimido. Para determinação do perfil de dissolução, nos tempos 5, 15, 30, 45, 60 e 90 minutos foram retiradas alíquotas de $1 \mathrm{~mL}$ para quantificação, com reposição do meio nas cubas, a fim de manter a estabilidade do sistema. As alíquotas foram diluídas em balões volumétricos de $10 \mathrm{~mL}$ com a fase móvel específica para o teste e submetidas a banho de ultrassom por 10 min para remoção de possíveis bolhas que pudessem comprometer a análise $(14,15)$. As amostras foram filtradas em filtros de membrana PTFE e analisadas por CLAE-UV. Os resultados obtidos foram analisados para avaliar se a tolerância preconizada na monografia da nimesulida (no mínimo, 80\% (Q) da quantidade declarada de $\mathrm{C}_{13} \mathrm{H}_{12} \mathrm{~N}_{2} \mathrm{O}_{5} \mathrm{~S}$ se dissolvem em $45 \mathrm{mi}$ nutos) foi atingida $(14,15)$.

Doseamento. O doseamento foi realizado de acordo com o método B descrito na monografia da nimesulida. Dessa forma, foram preparadas soluções-amostra para cada medicamento e padrão SQR. As soluções das amostras foram preparadas a partir da pesagem e pulverização de 20 comprimidos. Dessa massa, foi retirada uma quantidade de pó equivalente a $50 \mathrm{mg}$ de nimesulida e transferida para balão volumétrico de $100 \mathrm{~mL}$ contendo $60 \mathrm{~mL}$ da fase móvel.

Essa solução foi submetida a agitação mecânica durante 40 min e depois, o volume, correspondente ao balão, foi completado com a fase móvel. De posse dessa solução, uma alíquota de $2 \mathrm{~mL}$ foi tomada a qual foi diluída em $50 \mathrm{~mL}$ de fase móvel, para obtenção de outra solução, com concentração de $20 \mu \mathrm{g} /$ $\mathrm{mL}$, conforme preconizado. Logo após, a solução foi filtrada através de filtro de membrana PFTE para remoção dos excipientes e então $20 \mu \mathrm{L}$ foram injetados no cromatógrafo para mensuração das áreas sob os picos da amostra de Nisulid $^{\circledR}$ e Maxulid $^{\circledR}$ (15).

Para o preparo da solução padrão, $50 \mathrm{mg}$ de nimesulida SQR foram diluídos em $50 \mathrm{~mL}$ de fase móvel e submetida à banho de ultrassom por 10 min. Em seguida, foi tomada uma alíquota de $1 \mathrm{~mL}$ que novamente foi diluída em $50 \mathrm{~mL}$ de fase móvel para obter também a concentração de $20 \mu \mathrm{g} / \mathrm{mL}$, e então levada a banho de ultrassom por mais 10 $\mathrm{min})$. Por fim, essa solução foi filtrada com filtros de membrana PTFE antes das análises por CLAE (15).

\section{RESULTADO E DISCUSSÃO}

Os ensaios de qualidade têm a finalidade de assegurar que os medicamentos testados possuem realmente as propriedades ditas e se estão em conformidade com suas especificações. Para tal, são utilizadas metodologias analíticas apropriadas, de modo a assegurar que os produtos tenham sua qualidade comprovada antes de serem dispensados ao paciente (17).

De acordo com a RDC No 301, de 21 de agosto de 2019 da Anvisa, o controle da qualidade é obrigatório nas indústrias farmacêuticas e faz parte das Boas Práticas de Fabricação (BPF). Desse modo, assegura que os produtos só sejam liberados para comercialização depois que a qualidade dos mesmos seja comprovada, garantindo assim a segurança do seu uso (18), o que justifica a motivação do presente estudo em avaliar se todas as metodologias previstas nos compêndios oficiais vigentes, asseguram a qualidade e segurança dos novos medicamentos que usam excipientes com características complexantes como as ciclodextrinas.

Determinação de peso. $O$ teste de determinação de peso pode ser empregado em formas farmacêuticas sólidas e semissólidas e tem por finalidade analisar a uniformidade de peso entre unidades de um mesmo lote (14).

Os medicamentos em estudo apresentaram bons resultados, ou seja, dentro dos limites estabelecidos pela FB. Como pode ser observado na Tabela 1, Nisulid ${ }^{\circledR}$ apresentou peso médio de 0,405 g e, de acordo com a classificação da Farmacopeia, para comprimidos não revestidos com peso de $0,250 \mathrm{~g}$ ou mais, seu limite de variação de peso aceitável é de $\pm 5,0 \%$ (limite inferior de $0,385 \mathrm{~g}$ e limite superior de 0,425 g). Portanto, nenhuma unidade se apresentou fora do limite de variação de peso (14). 
Na Tabela 1 também é possível observar os dados relativos aos pesos dos comprimidos de Maxsulid $^{\circledR}$. O resultado do peso médio foi de $0,739 \mathrm{~g}$, apresentando, portanto, um critério de variação de peso também de $\pm 5 \%$ (tendo como limite inferior $0,703 \mathrm{~g}$ e superior de $0,777 \mathrm{~g}$ ). Desse modo, nenhuma unidade ficou fora do limite de variação (14).

Tabela 1. Determinação de peso nas amostras analisadas $(n=20)$.

\begin{tabular}{|l|c|c|}
\hline & Nisulid & Maxsulid $^{\circ}$ \\
\hline $\begin{array}{l}\text { Intervalo de pesos } \\
\text { individuais }\end{array}$ & $0,398-0,408 \mathrm{~g}$ & $0,720-0,746 \mathrm{~g}$ \\
\hline $\begin{array}{l}\text { Peso médio } \pm \mathrm{DP} \\
\text { (Variação de peso } \\
\text { aceitável } \pm 5,0 \%)\end{array}$ & $\begin{array}{c}0,405 \pm 0,002 \mathrm{~g} \\
(0,385-0,425 \mathrm{~g})\end{array}$ & $\begin{array}{c}0,739 \pm 0,007 \mathrm{~g} \\
(0,703-0,777 \mathrm{~g})\end{array}$ \\
\hline $\begin{array}{l}\mathrm{N}^{\circ} \text { de unidades fora da } \\
\text { taxa de variação }\left(\mathrm{N}^{\circ}\right. \\
\text { máximo permitido }=2)\end{array}$ & 0 & 0 \\
\hline
\end{tabular}

Portanto, ambos os medicamentos apresentaram sua determinação de peso dentro dos critérios de aceitação estabelecidos pela Farmacopeia Brasileira (14). Um resultado semelhante foi encontrado no estudo de Santos (2014), que realizou uma investigação da qualidade físico-química de comprimidos similares e genéricos de nimesulida 100 mg. A autora, que também utilizou a metodologia da FB, relatou obtenção de peso médio de $0,4037 \mathrm{~g}$ com uma variação mínima de $0,04 \%$ e máxima de $0,87 \%(1)$.

Friabilidade. De modo geral, comprimidos estão sujeitos a impactos físicos desde seu processo de produção até o manuseio pelo paciente. Dessa forma, o teste de friabilidade torna-se um ensaio de suma importância, visto que comprimidos com qualidade devem resistir a essas ações e permanecerem intactos até sua administração. $\mathrm{O}$ teste de friabilidade prevê condições de abrasão durante as operações unitárias de revestimento, empacotamento e transporte (1).

$\mathrm{Na}$ Tabela 2 estão expostos os resultados de ambos os medicamentos frente a esse teste de qualidade. Levando em consideração a diferença entre o peso inicial dos comprimidos e o final, após ação mecânica do aparelho, para o Nisulid $^{\circledR}$ a porcenta- gem de pó perdido foi de $1,37 \%$, ou seja, dentro do limite de aceitação, que é 1,5\% (14).

Para os comprimidos de Maxsulid ${ }^{\circledR}$, a diferença entre o peso inicial e final representou uma perda de $0,76 \%$, resultado dentro do limite de $\leq 1,5 \%$ de pó perdido (14).

Tabela 2. Teste de friabilidade de comprimidos Nisulid $^{\circledR} n=20$ e Maxsulid $\left.{ }^{\circledR} n=10\right)$.*

\begin{tabular}{|c|c|c|}
\hline & Nisulid $^{\circ}$ & Maxsulid $^{\circ}$ \\
\hline Pesos iniciais $(\mathrm{g}) \pm \mathrm{DP}$ & $8,102 \pm 0,002$ & $7,392 \pm 0,011$ \\
\hline Pesos finais $(\mathrm{g}) \pm \mathrm{DP}$ & $7,991 \pm 0,002$ & $7,337 \pm 0,011$ \\
\hline Perda (g) & 0,111 & 0,055 \\
\hline $\begin{array}{l}\text { Perda percentual } \\
\text { (valor aceitável } \leq 1,5 \% \text { ) }\end{array}$ & $1,37 \%$ & $0,76 \%$ \\
\hline
\end{tabular}

Dureza. Para esse teste, os resultados são apresentados na Tabela 3 em Newtons (N). A dureza de um comprimido indica a resistência mecânica contra rupturas e esmagamentos e segundo a Farmacopeia Brasileira, o resultado desse teste é apenas informativo, não possuindo um intervalo estabelecido para aprovar ou reprovar um medicamento (14).

Os comprimidos de Maxsulid $^{\circledR}$ apresentaram uma média de dureza aproximadamente 4,5 vezes maior do que os de Nisulid $^{\circledR}$. Vale ressaltar que os comprimidos de Maxsulid ${ }^{\circledR}$ apresentam também um tamanho superior, e os comprimidos maiores necessitam de mais força para serem quebrados, apresentando-se mais duros do que os comprimidos menores. Além do tamanho do comprimido, a concentração utilizada de lubrificantes, tempo e força de compressão, e até mesmo o tipo de punção utilizado podem interferir na dureza de um comprimido (19).

Tabela 3. Intervalos de dureza dos comprimidos de Nisulid $^{\circledR}$ e Maxsulid $^{\circledR}$.

\begin{tabular}{|l|c|c|}
\hline & Nisulid $^{\circ}$ & Maxsulid $^{\circ}$ \\
\hline Dureza & $49,0-59,8 \mathrm{~N}$ & $197,0-257 \mathrm{~N}$ \\
\hline Dureza média \pm DP & $54,39 \pm 3,69 \mathrm{~N}$ & $245,28 \pm 30,52 \mathrm{~N}$ \\
\hline
\end{tabular}


No estudo de Santos (2014), os valores de dureza obtidos foram relacionados com os resultados do teste de friabilidade, visto que os comprimidos que apresentaram uma menor dureza também foram os mais friáveis. $\mathrm{O}$ mesmo se repetiu no presente estudo, onde os comprimidos de Nisulid $^{\circledR}$ tiveram seus resultados para dureza e friabilidade inferiores aos comprimidos de $\operatorname{Maxsulid}^{\circledR}$ (1).

Bueno e cols. (2010) avaliaram a qualidade de quatro especialidades farmacêuticas contendo hidroclorotiazida, mas seguiram como referência o valor estabelecido para o teste de dureza descrito na Farmacopeia Brasileira 4a edição (1988) $\geq 30 \mathrm{~N}$, e por isso, duas amostras foram reprovadas. Se esse parâmetro ainda fosse previsto na $\mathrm{FB} 6^{\mathrm{a}}$ edição, ambos os medicamentos estariam aprovados, visto que mostraram uma dureza média maior que $30 \mathrm{~N}$ (20).

Desintegração. O teste de desintegração avalia o tempo necessário até a desintegração total dos comprimidos ou cápsulas no aparelho desintegrador em condições experimentais descritas na Farmacopeia Brasileira. O limite de tempo especificado para comprimidos não revestidos é de 30 min (14).

Os comprimidos de Nisulid $^{\circledR}$ desintegraram completamente em 3 min e $67 \mathrm{~s}$, e os comprimidos de Maxsulid ${ }^{\circledR}$ em 4 min e 55 s, tempos de desintegração dentro do preconizado pela Farmacopeia Brasileira (14).

Podem ser atribuídos, como variáveis à desintegração de um medicamento, porosidade, solubilidade, área superficial, polimorfismo, fatores de pré-formulação e formulação, como o uso de excipientes, pressão e tempo de compressão, além de fatores relacionados ao meio de reação, como $\mathrm{pH}$, temperatura, agitação, presença de adsorventes e tensão superficial (21).

Visto que a área de superfície de um comprimido tem influência em sua desintegração e, o comprimido de Nisulid $^{\circledR}$ apresenta um tamanho menor comparado ao de Maxsulid ${ }^{\circledR}$, a diferença no tempo obtida nesse teste pode ser atribuída a este fator.

Há diferenças no número dos excipientes utilizados em cada medicamento, sendo possível observar que o Nisulid $^{\circledR}$ possui um desintegrante a mais (hiprolose), comparado com o Maxsulid ${ }^{\circledR}$ (22,
23). No estudo conduzido por Santos (2014), não foram observadas diferenças expressivas na formulação dos medicamentos em teste; assim, a autora levantou uma hipótese relacionando a diferença do tempo de desintegração dos medicamentos em teste com a concentração utilizada de cada excipiente, e não com o número deles (1).

Um agente desintegrante faz com que o comprimido se desintegre em fragmentos menores a fim de obter uma maior área de superfície para ser dissolvido e absorvido (1). Por isso, atribuir à composição do Nisulid ${ }^{\circledR}$ ao menor tempo de desintegração também é uma hipótese válida para explicar os resultados obtidos na presente investigação.

Análise por Cromatografia líquida de alta eficiência (CLAE). O cromatograma exemplo obtido pelas análises por CLAE está apresentado na Figura 1, no qual pode ser observado que o método farmacopeico foi reproduzido com sucesso garantindo assim a seletividade do método. Não foram observadas interferências externas, a exemplo do diluente, fase móvel, impurezas ou produtos de degradação, conforme é esperado de acordo com a RDC 166/2017 (16). A partir do cromatograma, pode ser observado que o tempo de retenção da nimesulida foi de aproximadamente sete minutos e que a pureza cromatográfica foi demonstrada por meio do sinal do analito.

Rao e cols. (2005) conduziram uma extensa revisão da literatura acerca dos métodos analíticos mais usados para quantificação de inibidores da ciclooxigenase-2, classe da qual faz parte a nimesulida. Neste estudo, os autores relataram que CLAE foi a técnica analítica mais utilizada, seguida pela espectrofotometria no UV e visível. Porém, a partir desse mesmo estudo, os autores observaram que existia uma grande variação nas condições cromatográficas utilizadas, desde o tipo de coluna, $\mathrm{pH}$ e composição da fase móvel, até comprimento de onda para detecção do analito (24).

Ruela e cols. (2009), embasados no estudo acima, variaram as condições cromatográficas e adaptaram o método para a determinação da nimesulida. A fase móvel foi modificada para acetonitrila e tampão fosfato $(55: 45 \mathrm{v} / \mathrm{v}), \mathrm{o} \mathrm{pH}$ para 6,0 e o comprimento de onda para detecção para 300 nm. Como resultado, obtiveram resultados mais rá- 
Figura 1. Cromatograma da nimesulida obtido a partir da cromatografia líquida de alta eficiência (CLAE-UV, $220 \mathrm{~nm}$ ).

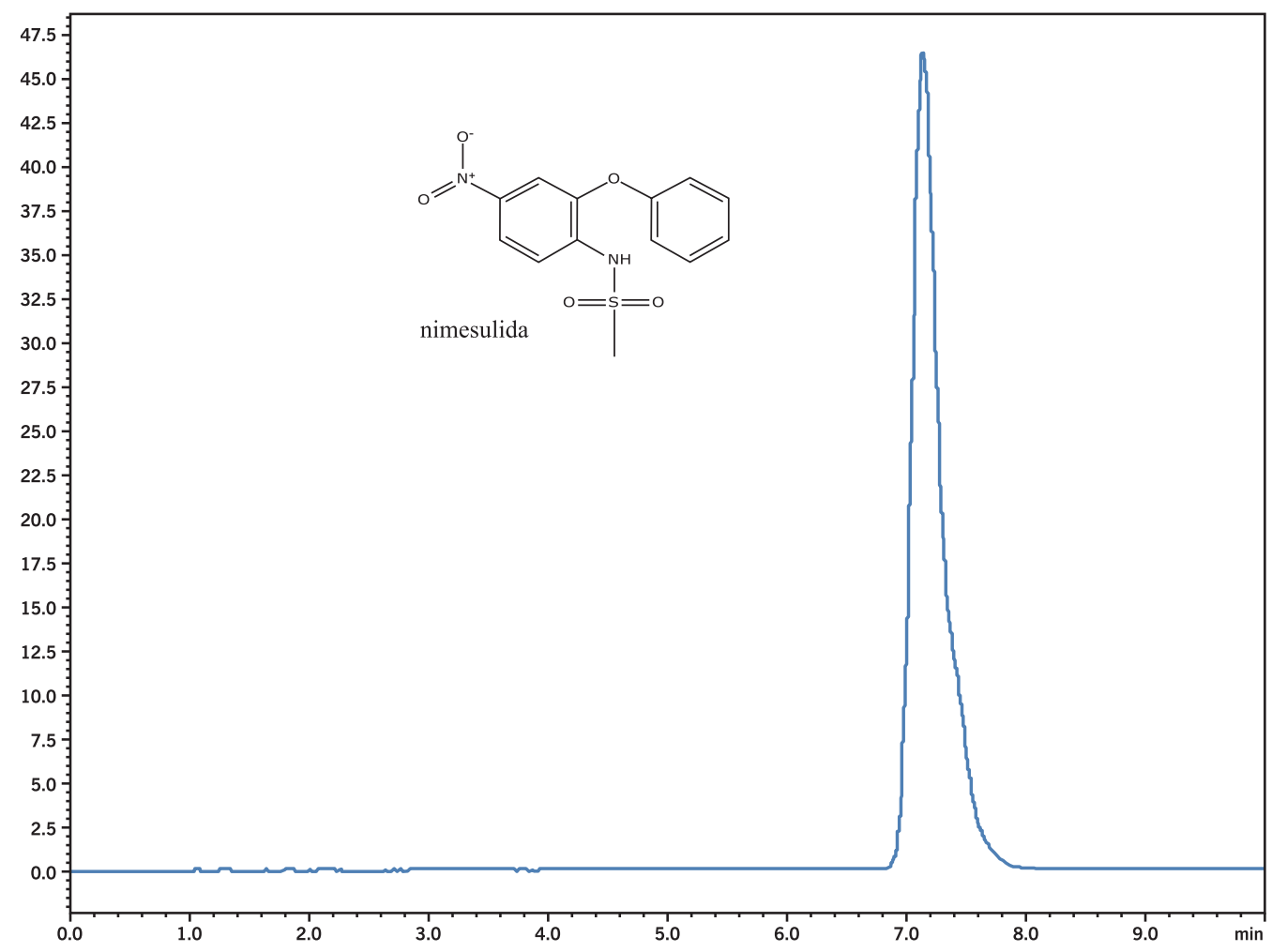

Figura 2. Perfil de dissolução dos comprimidos de Nisulid $^{\circledR}$ e Maxsulid $^{\circledR}$. Resultados expressos como média e barras de erros representam os desvios- padrão.

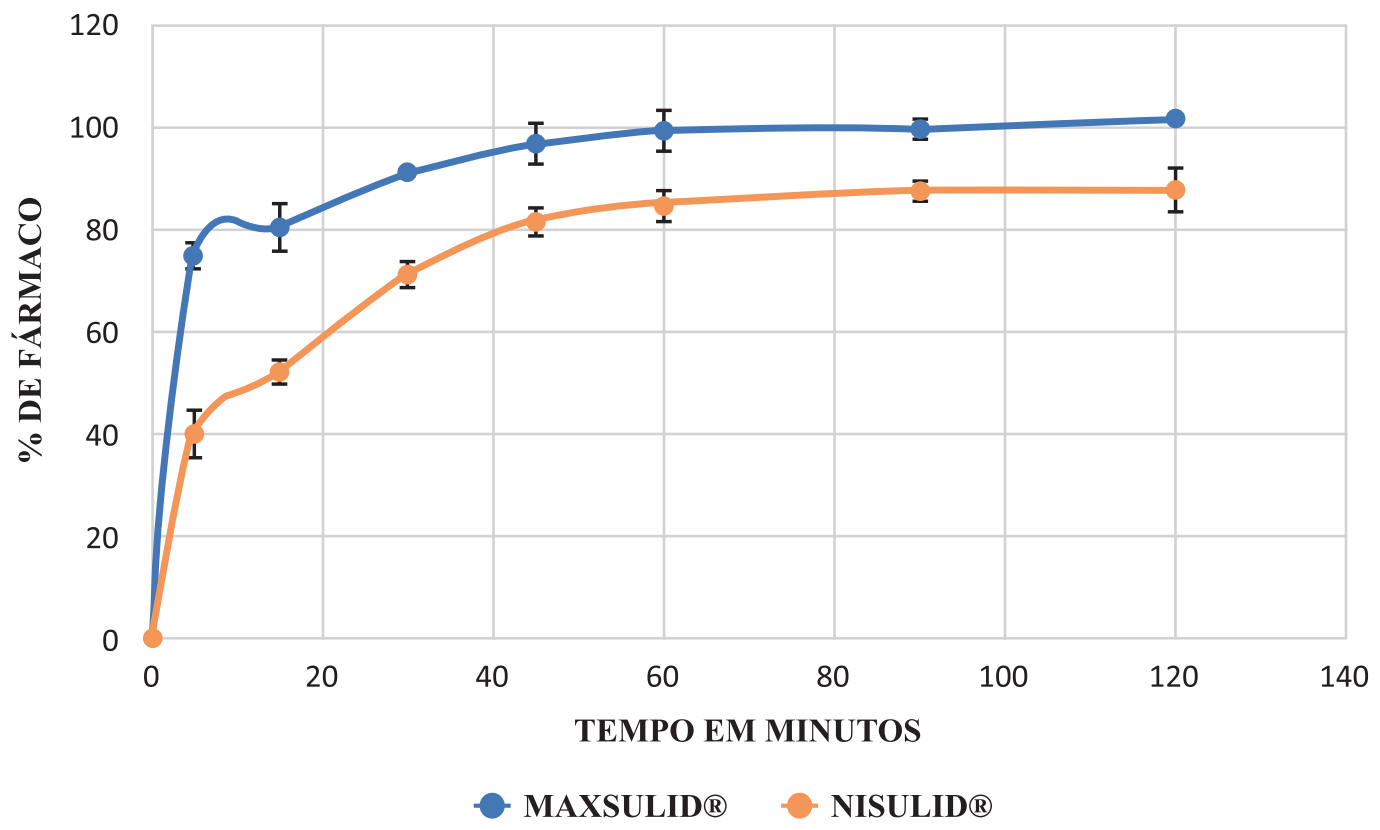


pidos, visto que o pico cromatográfico foi obtido antes mesmo de completar três minutos de corrida. No presente estudo, seguindo as recomendações farmacopeicas, o sinal analítico da nimesulida foi detectado por volta de sete minutos (25).

Dissolução. O ensaio de dissolução permite determinar a quantidade de substância ativa dissolvida do medicamento em teste, quando colocado em aparelhagem e condições experimentais específicas (14). Um dos fatores que influenciam a atividade de um fármaco é sua concentração nos fluidos biológicos. Inicialmente, essa concentração é determinada pela desintegração e dissolução do ativo, presente em uma forma farmacêutica sólida, seguida de sua permeação nas membranas celulares. Assim, a dissolução de um medicamento está diretamente relacionada com a biodisponibilidade do fármaco (26).

O perfil de dissolução (Figura 2) foi calculado a partir da equação obtida pela curva de calibração da nimesulida ( $y=13472 x-42233 ; r=0,9997)$, onde foram utilizadas as áreas dos picos obtidos para cada medicamento nos tempos de coleta, a fim de obter a concentração experimental, e os resultados foram expressos em quantidade dissolvida (\%).

Com apenas 5 min. de teste, Maxsulid ${ }^{\circledR}$ apresentava $75 \%$ dissolvido no meio, enquanto Nisu$\operatorname{lid}^{\circledR}$, apenas $40 \%$. Para todos os outros tempos de coleta, Maxsulid ${ }^{\circledR}$ continuou sendo o medicamento com o conteúdo mais dissolvido. Ao final do teste, em 120 min, Maxsulid ${ }^{\circledR}$ foi totalmente dissolvido. Por outro lado, o Nisulid ${ }^{\circledR}$ atingiu a faixa de $87 \%$. Esse aumento da solubilidade, que culminou em uma maior dissolução do fármaco, pode ser atribuído a complexação com a $\beta$-CD.

Pereira e cols (2016) conduziram um estudo de desenvolvimento e validação de metodologia analítica para ensaios de dissolução de comprimidos de nimesulida- $\beta$-CD $400 \mathrm{mg}$. Nesse estudo, os autores testaram diversas condições experimentais e observaram que a duração do teste de dissolução poderia ser de 60 minutos, tendo como meio de dissolução $900 \mathrm{~mL}$ de solução aquosa de laurilsulfato de sódio $1 \%(\mathrm{p} / \mathrm{v})$ sob agitação de $100 \mathrm{rpm}$, usando aparato pá (27).

Esse estudo é interessante, pois embora alguns parâmetros sejam diferentes daqueles preconizados pela FB, 99,08\% de fármaco foi liberado em 60 min, enquanto no presente estudo, houve liberação de $99,52 \%$ no mesmo tempo, o que mostra que, para teste de dissolução, bem como os demais ensaios avaliados acima, a FB possui metodologia adequada para garantir qualidade e segurança dos medicamentos registrados, inclusive aqueles que possuem agentes complexantes como a ciclodextrina.

Auda (2014), em um estudo de caracterização físico-química, solubilidade e estudos biológicos de complexos de inclusão de nimesulida/metil- $\beta$ -CD, realizou uma análise dos cristais por difratometria de raios $\mathrm{X}$ e concluiu que o complexo de inclusão diminuiu a cristalinidade da nimesulida. Desse modo, a diminuição na cristalinidade do medicamento na complexação pode ser um fator contribuinte para o aumento da solubilidade, e, portanto, de sua dissolução (7).

Essa melhora na dissolução da nimesulida complexada em $\beta-C D\left(\right.$ Maxulid $^{\circledR}$ ) também foi observada no estudo de Nalluri e cols. (2003). Os autores realizaram a caracterização físico-química e determinaram as propriedades de dissolução da nimesulida complexada ou não em $\beta$-CD. Os resultados, de forma similar ao presente estudo, mostraram que a nimesulida complexada mostrou um perfil de dissolução superior quando comparada à não complexada. Os autores atribuíram esse fenômeno à maior interação interpartícula da nimesulida com $\beta-C D$, quando comparada a formulação que fez uso de excipientes desprovidos de capacidade complexante (28).

Visto que a solubilidade é um fator importante para a liberação e absorção de um fármaco e, por isso, influencia em sua biodisponibilidade, as CD melhoram esse parâmetro (29). Outros estudos envolvendo AINE complexados em CDs também mostraram esta melhora da solubilidade em relação ao fármaco não complexado, a exemplo do meloxicam- $\gamma-C D$, piroxicam- $\beta-C D$ indometacina- $\beta C D$ (30-32).

O estudo de Scarpignato (2013) mostrou que quando medicamentos complexados são administrados oralmente, como no caso dos AINE, o uso primário das $\mathrm{CD}$ melhora solubilidade, taxa de dissolução e estabilidade do fármaco no trato gastrintestinal, de modo a reduzir o tempo de contato com 
a mucosa, o que culmina em uma maior absorção gástrica com mínimas reações adversas em relação aos medicamentos não complexados (31).

Doseamento. O doseamento é um teste de quantificação de grande importância na garantia de segurança, eficácia e qualidade dos medicamentos, pois determina se a dosagem indicada na embalagem do fabricante condiz com a de fato encontrada no medicamento (33).
O teor de nimesulida foi calculado a partir das áreas obtidas do cromatograma por CLAE. Esses valores foram aplicados na equação da reta, obtida por meio da curva de calibração da nimesulida $(y=13472 x-42233 ; r=0,9997)$. Os teores percentuais encontrados estão apresentados na Tabela 4, onde é possível perceber que o Nisulid ${ }^{\circledR}$, encontra-se dentro das especificações descritas na $6^{\text {a }}$ edição da Farmacopeia Brasileira (15).

Tabela 4. Doseamento, expresso em porcentagem do padrão de nimesulida SQR, Nisulid ${ }^{\circledR}$ e Maxsulid $^{\circledR}$ $(\mathrm{n}=3)$.

\begin{tabular}{|c|c|c|c|}
\hline & Nimesulida SQR & Nisulid $^{\circ}$ & Maxsulid $^{\circ}$ \\
\hline Especificação & $98,5 \%-101,5 \%$ & $95,0 \%-105,0 \%$ & $95,0 \%-105,0 \%$ \\
\hline \% médio de fármaco & $99,14 \%$ & $98,66 \%$ & $50,03 \%$ \\
\hline Desvio padrão & $2,50 \%$ & $5,51 \%$ & $2,24 \%$ \\
\hline
\end{tabular}

No entanto, Maxsulid ${ }^{\circledR}$, que apresenta a $\beta$-CD como excipiente capaz de formar complexos de inclusão com o fármaco, ficou muito abaixo do esperado. A partir deste resultado, pode ser inferido que a metodologia da Farmacopeia Brasileira 6 edição (2019) ainda não está adequada para doseamento correto de medicamentos que possuem tecnologias mais recentes, como complexos de inclusão, na sua composição, o que mostra que os métodos vigentes não se aplicam para extração eficiente do fármaco complexado. Outra limitação reside na falta de informações provenientes do fabricante acerca do método usado na complexação, razão fármaco:ciclodextrina, eficiência de complexação no referido lote, tampouco constante de complexação, o que impossibilita o cálculo acurado do doseamento.

A metodologia da $6^{\text {a }}$ edição da Farmacopeia Brasileira para o doseamento da nimesulida através da cromatografia líquida de alta eficiência preconiza

"Solução amostra: pesar e pulverizar 20 comprimidos. Transferir quantidade do pó equivalente a $50 \mathrm{mg}$ de nimesulida para balão volumétrico de $100 \mathrm{~mL}$, adicionar $60 \mathrm{~mL}$ da Fase móvel e agitar mecanicamente por 40 minutos. Completar o volume com Fase móvel e filtrar.
Diluir, sucessivamente, em Fase móvel, de modo a obter solução a $20 \mu \mathrm{g} / \mathrm{mL}$ " (Farmacopeia Brasileira, $\sigma^{a}$ edição, 2019) (15).

No presente estudo, a metodologia acima referida foi seguida para ambos os medicamentos. De acordo com os dados apresentados na Tabela 4, o método foi reprodutível para o Nisulid $^{\circledR,}$ e a agitação mecânica foi suficiente para extrair o fármaco da associação com os excipientes. Todavia, não se aplica para o Maxulid ${ }^{\circledR}$, para o qual, claramente, o fármaco complexado não foi eficientemente extraído da cavidade da $\beta$-CD pelo método descrito na Farmacopeia.

No estudo de Nalluri e cols. (2007), os autores investigaram a formação de comprimidos a partir de sistemas binários de nimesulida- $\beta C D$ (razão molar 1:2), obtidos pelos métodos da pasta ou co-evaporação) e meloxicam- $\gamma C D$ (razão molar 1:1 e 1:2, método da pasta). Para o doseamento dos fármacos, os autores usaram seis comprimidos pulverizados equivalente a $10 \mathrm{mg}$ de nimesulida ou meloxicam. Cada fármaco foi dissolvido em um balão volumétrico de $100 \mathrm{~mL}$, contendo $25 \mathrm{~mL}$ de metanol. A solução resultante foi submetida ao vortex durante cinco minutos e então, o menisco do balão foi aferido com metanol. A solução foi filtrada em filtro $0,45 \mu \mathrm{m}$ e submetida à quantificação. 
Nesta análise, o teor de nimesulida foi de $100 \%$ $\pm 5 \%$ (30).

A partir desse estudo é possível observar a diferença que uma extração realizada com vortex faz em relação à agitação mecânica, que é menos potente, na remoção do fármaco da cavidade da ciclodextrina. A agitação em vortex intensifica o processo de dispersão do solvente extrator e contribui para aumentar a superfície de contato entre o solvente extrator e o fármaco, que favorece a decomplexação da nimesulida e culmina em um doseamento adequado (34). Desse modo, dentre as metodologias farmacopeicas analisadas no presente estudo, o doseamento, na forma como consta na $\mathrm{FB}$, não está adequado para extração eficiente do fármaco para posterior quantificação, o que sugere que o baixo teor de $\operatorname{Maxsulid}^{\circledR}(50,03 \%)$, não está relacionado a desvio de qualidade (hipótese suportada pela aprovação do medicamento nos demais ensaios realizados) e sim, a uma limitação metodológica neste teste.
Essa é uma limitação importante e esperada do presente trabalho, uma vez que reforça ainda mais a necessidade de atualização das normas vigentes para quantificação do teor dos medicamentos recentemente lançados no mercado, com ou sem mecanismos envolvidos de liberação controlada. Mesmo que a CD seja considerada um excipiente que não conduza à liberação controlada de fármacos, a sua capacidade de formar complexos de inclusão altera a solubilidade aparente do fármaco, o que influencia diretamente no doseamento e, consequentemente, no perfil de liberação do mesmo, pois aumenta a biodisponibilidade do medicamento, conforme já elucidado.

Vale salientar a necessidade de atualização da FB (2019) para o doseamento de medicamentos novos que incluem sistemas de liberação tais como lipossomas, nanopartículas e ciclodextrinas. O Quadro 1 mostra medicamentos contendo ciclodextrina na sua composição com registro na Anvisa anterior à publicação da última edição da FB (2019).

Quadro 1. Medicamentos registrados na Agência Nacional de Vigilância Sanitária (Anvisa), contendo ciclodextrina na sua composição.

\begin{tabular}{|c|c|c|c|}
\hline Nome comercial & IFA & Empresa detentora do registro & Ano do registro \\
\hline Maxulid $^{\odot}$ & nimesulida & $\begin{array}{c}\text { Cosmed Ind de Cosméticos e } \\
\text { Medicamentos S.A. }\end{array}$ & 2011 \\
\hline Cicladol $^{\oplus}$ & piroxicam & Chiesi Farmacêutica LTDA & 2000 \\
\hline Brexin $^{\bullet}$ & piroxicam & Laboratório Gross S. A. & 2002 \\
\hline Yaz $^{\odot}$ & etinilestradiol & Bayer S. A. & 2010 \\
\hline
\end{tabular}

Dados disponíveis em http://www.smerp.com.br/anvisa/. Acesso 12/07/2020 às 18:26h.

Savaşer e cols. (2004) validaram um método para determinar o complexo piroxicam- $\beta-C D$ em comprimidos e no plasma humano usando CLAE. Foram obtidos resultados positivos para o doseamento, porém os autores tinham conhecimento da razão molar da complexação do medicamento, e por isso realizaram os testes sem interferência de excipientes (10).

Camargo e cols. (2013) conduziram um estudo de doseamento de sulfadiazina de prata complexada com $\beta-\mathrm{CD}$, por metodologia descrita em um estudo publicado no Iranian Journal of Phar- maceutical Research e as amostras analisadas de acordo com a Farmacopeia Americana de 2007, também por CLAE-UV (254 nm). Como resultado, obtiveram baixo teor do complexo, o qual variou de $0,16 \%$ a $0,23 \%$. Entretanto, mesmo com baixo teor, o fármaco foi capaz de causar uma inibição positiva frente aos microrganismos testados (35), o que sugeriu que, embora o fármaco não tenha sido eficientemente quantificado, como no estudo presente, ainda assim, manteve o seu efeito terapêutico, o que deve acontecer com a nimesulida- $\beta C D$. 


\section{CONCLUSÃO}

Os resultados obtidos nesse estudo comparativo evidenciaram a qualidade dos medicamentos de ambas as marcas, sugerindo que possuem qualidade adequada para uso terapêutico, sendo aprovados em quase a totalidade dos ensaios. Todavia, Maxsulid $^{\mathbb{B}}$ apresentou baixo teor no ensaio de doseamento, o que foi atribuído à falha metodológica por falta de atualização da FB $6^{\mathrm{a}}$ edição, bem como da indisponibilidade de informações técnicas inerentes ao lote do medicamento. Esta limitação reforça a necessidade de atualização dos protocolos analíticos previstos para os medicamentos registrados como novos, detentores de tecnologias farmacêuticas envolvidas na produção dos medica- mentos recentemente lançados no mercado, como uma estratégia segura de confirmar a eficácia dos medicamentos dispensados à população.

\section{AGRADECIMENTOS}

Os autores agradecem à PlenaPharma - Farmácia de Manipulação pela doação da nimesulida SQR para execução do teste de doseamento e curva de calibração. Igualmente à Universidade Federal de Sergipe e toda equipe do Laboratório de Ensaios Farmacêuticos e Toxicidade da UFS, pela possibilidade de realização deste trabalho, bem como as agências de fomento como FINEP e FAPITEC-SE que viabilizaram a aquisição dos equipamentos usados na presente pesquisa.

\section{REFERÊNCIAS}

1. Santos BWL. Estudo da qualidade físico-química de comprimidos similares e genéricos de nimesulida 100 mg e validação de metodologia analítica para cápsulas magistrais [dissertação]. Brasília: Faculdade de Ciências da Saúde, Universidade de Brasília. 2014.

2. Bernareggi, A. The Pharmacokinetic Profile of Nimesulide in Healthy Volunteers. Drugs. 1993; 46:64-72. DOI: 10.2165/00003495-199300461-00013.

3. Amidon; GL; Lennernäs H; Shah VP; Crison JR. A theoretical basis for a biopharmaceutic drug classification: the correlation of in vitro drug product dissolution and in vivo bioavailability. Pharm Res. 1995;12(3):413-420. DOI: 10.1023/A:1016212804288.

4. Cunha-Filho MSS; Sá-Barreto LCL. Utilização de ciclodextrinas na formação de complexos de inclusão de interesse farmacêutico. Rev. Ciênc. Farm. Básica. 2007; 28(1): 1-9.

5. Guedes FL; Alves GMC; Santos FLA; Lima LF; Rolim LA; Rolim Neto; PJR. Ciclodextrinas: como adjuvante tecnológico para melhorar a biodisponibilidade de fármacos. Rev. Bras. Farm. 2008;89(3):220-225.

6. Venturini CDG; Nicolini J; Machado C; Machado VG. Propriedades e aplicações recentes das ciclodextrinas. Quím Nova. 2008; 31(2):360-368. DOI: 10.1590/ S0100-40422008000200032.

7. Auda SH. Nimesulide/methyl $\beta$-cyclodextrin inclusion complexes: Physicochemical characterization, solubility, dissolution, and biological studies. Drug Develop Res Rev. 2014; 75(2):68-75. DOI: 10.1002 / ddr.21156.
8. Gidwani B; Vyas A. A Comprehensive Review on Cyclodextrin-Based Carriers for Delivery of Chemotherapeutic Cytotoxic Anticancer Drugs. Biomed Res Int. 2015; DOI: 10.1155/2015/198268.

9. Schwarz DH; Engelke A; Wenz G. Solubilizing steroidal drugs by $\beta$-cyclodextrin derivatives. Int J Pharm. 2017;531(2):559-567. DOI: 10.1016/j. ijpharm.2017.07.046.

10. Savaşer; A; Karatas A; Özkan Y; Yüksel N; Özkan SA; Baykara T. Validated LC determination of the piroxicam$\beta$-cyclodextrin inclusion complex in tablets and in human plasma. Chromatographia. 2004; 59(9-10):555-560. DOI: $10.1365 / \mathrm{s} 10337-004-0256-2$.

11. Keleş; GT; Topcu I; Ekici Z; Yentür A. Evaluation of piroxicam- $\beta$-cyclodextrin as a preemptive analgesic in functional endoscopic sinus surgery. Braz $\mathrm{J}$ Med Biol. 2010;43(8):806-811. DOI: 10.1590/S0100$879 \times 2010007500063$.

12. Yameógo JBG; Mazeta R; Wouessidjewea D; Choisnarda L; Godin-Ribuotd D; Putauxe JL; Semdéc R; Gèzeet A. Pharmacokinetic study of intravenously administered artemisinin-loaded surface-decorated amphiphilic $\gamma$-cyclodextrin nanoparticles. Mater Sci Eng C Biol Appl. 2019;106:1-9. DOI: 10.1016/j.msec. 2019.110281.

13. Silva CB; Hauser-Davis RA; Silva ALO; Moreira JC. Desafios ao controle da qualidade de medicamentos no Brasil. Cad. Saúde Colet. 2017;25(3):362-370. DOI: 10.1590/1414-462x201700030075. 
14. BRASIL. Farmacopeia Brasileira, volume 01. 6 $6^{\mathrm{a}}$ Ed. Brasília: Agência Nacional de Vigilância Sanitária. 2019.

15. BRASIL. Farmacopeia Brasileira, volume 02 Monografias, insumos farmacêuticos e especialidades. $6^{\text {a }}$ Ed. Brasília: Agência Nacional de Vigilância Sanitária. 2019.

16. BRASIL. Resolução RDC $\mathrm{N}^{\circ} 166$, de 24 de julho de 2017. Guia para validação de métodos analíticos. Agência Nacional de Vigilância Sanitária. Diário Oficial da União, no 141, 25 de julho de 2017.

17. Silva FJ; Rodrigues MH; Freitas TM; Pinto MV. Controle de qualidade físico-químico de comprimidos de ibuprofeno $300 \mathrm{mg}$. Rev. Facul. Montes Belos. 2014;7(1):151-162.

18. BRASIL. Resolução RDC No 301, de 21 de agosto de 2019. Dispõe sobre as Boas Práticas de Fabricação de Medicamentos. Agência Nacional de Vigilância Sanitária. Diário Oficial da União, $\mathrm{n}^{\circ}$ 162, 22 de agosto de 2019.

19. Lachman L; Lieberman HA; Kanig JL. Teoria e prática na indústria farmacêutica. $2^{\mathrm{a}}$ ed. Lisboa: Fundação Calouste Gulbenkian. 2001.

20. Bueno CS; Weber D; Moreira AC. Avaliação da qualidade de quatro especialidades farmacêuticas contendo hidroclorotiazida. Rev. Bras. Farm. 2010;91(3):126-132.

21. Santos-Júnior MC; Caetité-Júnior E; Santos-Júnior AF. Infuência de parâmetros físico-químicos sobre o tempo de desintegração de comprimidos de paracetamol. Infarma - Ciênc Farmac. 2013;19(3-4):11-16.

22. NISULID®: nimesulida. Dr. Wilson R. Farias. Guarulhos/SP: Aché, (2018). Bula de remédio.

23. MAXSULID®: nimesulida betaciclodextrina. Fernando Costa Oliveira. Anápolis/GO: Farmasa (2018). Bula de remédio.

24. Rao RN; Meena S; Rao AR. An overview of the recent developments in analytical methodologies for determination of COX-2 inhibitors in bulk drugs, pharmaceuticals and biological matrices. J. Pharm. Biomed. Anal. 2005; 39(3-4):349-363. DOI: 10.1016/j. jpba.2005.03.040.

25. Ruela ALM; Araújo MB; Pereira GR. Development and validation of a rapid analytical method by HPLC for determination of nimesulide in release studies. Quim Nova. 2009;32(1):165-168. DOI: 10.1590/S0100-40422 009000100031 .
26. Lavra ZMM. Incremento da solubilidade e da cinética de dissolução do fármaco Efavirenz através da obtenção de misturas binárias amorfas com matrizes poliméricas. [Tese]. Recife: Universidade Federal de Pernambuco. 2016.

27. Pereira C; Dias L; Oliveira G; Vila Verde G; Aquino; G. Development and Validation Dissolution Analytical Method of Nimesulide beta-Cyclodextrin $400 \mathrm{mg}$ Tablet. Orbital: Electron. J. Chem. 2016;8(5):300-306. DOI: 10.17807/orbital.v8i5.827.

28. Nalluri BN; Hayman AR; Becket G. Physicochemical characterization and dissolution properties of nimesulidecyclodextrin binary systems. AAPS PharmSciTech. 2003; 4(1):E2. DOI: 10.1208/pt040102.

29. Panchagnula R; Thomas NS. Biopharmaceutics and pharmacokinetics in drug research. Int. J. Pharm. 2000; 201:131-50. DOI: 10.1016/S0378-5173(00)00344-6.

30. Nalluri BN; Chowdary KPR; Kolapalli RV; Becket G; Crooks P. Tablet formulation studies on nimesulide and meloxicam-cyclodextrin binary systems. AAPS PharmSciTech. 2007; 8(2): article 36. DOI: 10.1208/ pt0802036.

31. Scarpignato C. Piroxicam- $\beta$-Cyclodextrin: A GI Safer Piroxicam. Curr Top Med Chem. 2013;20(19). DOI: 10.2174/09298673113209990115

32. Jambhekar S; Casella R; Maher T. The physicochemical characteristics and bioavailability of indomethacin from beta-cyclodextrin, hydroxyethyl-beta-cyclodextrin, and hydroxypropyl-beta-cyclodextrin complexes. Int J Pharm. 2004;270(1-2):149-166. DOI: 10.1016/j.ijpharm.2003. 10.012 .

33. Roveril FL; Faria LG; Ribeiro-Neto LM; Silva AM. Avaliação da aplicação de método espectrofotométrico para determinação do teor de ibuprofeno em diferentes formas farmacêuticas. Rev. Bras. Farm. 2012;93(2): 186-190.

34. Duarte JS; Dores EFGC; Villa RD. Microextração líquido-líquido dispersiva assistida por vortex e ultrassom aplicada à determinação de agrotóxicos triazinas, triazinonas e o triazol flutriafol em água. Quim Nova. 2016;39(8):925-931. DOI: 10.5935/01004042.20160110 .

35. Camargo FP; Raffin R; Rossato J; Silva IZ; Hoelzel SCSM. Preparação, caracterização, atividade antimicrobiana e simulação computacional do complexo de inclusão de sulfadiazina de prata com $\beta$-Ciclodextrina. RDS. 2013;14(1). DOI: 10.37779/nt.v14i1.1316. 